\title{
Rare complication of needleless mini-sling procedure: Case report
}

\author{
Mustafa Karalar, MD; Ibrahim Keles, MD; ${ }^{1}$ Serdar Unlu, MD; ${ }^{2}$ Hasmet Sarici, MD; ${ }^{-1}$ Mesut Kose, MD;2 \\ Cemil Ay, $M D^{\prime}$
}

'Department of Urology; ${ }^{2}$ Department of Gynecology; Afyon Kocatepe University, Afyonkarahisar, Turkey

Cite as: Can Urol Assoc J 2017;1 1 (3-4):E1 16-8. http://dx.doi.org/10.5489/cuaj.3245

Published online March 16, 2017

\section{Abstract}

The mini-sling procedure is a widely used, minimally invasive treatment for stress urinary incontinence. While bladder perforation and stone formation over the mesh is not an expected complication of the mini-sling procedure, in this case, we report on the management of bladder calculi formed over the mesh, which was passed through the bladder while applying the mini-sling procedure, and was eventually removed using holmium laser. Performing cystoscopy in patients with irritative and obstructive symptoms after the sling procedure will help confirm bladder perforation, and an endoscopic approach using holmium laser is an effective treatment.

\section{Introduction}

Stress urinary incontinence (SUI) is a common health problem. Conservative techniques, as well as pharmacological and surgical approaches, are used in the treatment of SUI. ${ }^{1}$ The aim of surgery is to correct the deficient urethral closure and to prevent incontinence.

Ulmsten et al described a minimally invasive technique, a tension-free vaginal tape (TVT) for SUI, in 1996 and it has become the gold standard procedure. ${ }^{2}$ In 2001, Delorme et al described the transobturator tape implantation (TOT) method to avoid the risk of bladder and bowel perforation and major vascular injuries that can occur with $\mathrm{TVT}^{3}{ }^{3}$ however, similar complications were observed. ${ }^{1}$ In 2006, a thirdgeneration of midurethral tapes - known as single-incision mini-slings - was introduced to reduce complications such as bladder perforation. ${ }^{1}$ The mini-sling procedure is a widely used, minimally invasive treatment for SUI; there are only a few reports on its complications. ${ }^{4}$

Bladder perforation and stone formation over the mesh are not expected complications of mini-sling procedures. ${ }^{5}$ Further, the technique of removing an eroded tape from the bladder remains controversial. ${ }^{6}$
In this case, we report on the management of bladder calculi formed over the mesh, which was passed through the bladder while applying the mini-sling procedure, and removed using holmium laser.

\section{Case report}

A 46-year-old woman was admitted to our urology clinic for pelvic pain, dysuria, recurrent urinary tract infections, urinary frequency, and urgency. She had a history of stress urinary incontinence, which had been treated with a minisling procedure using a needleless device. The procedure had been performed at a different hospital four months prior and her complaints began after the procedure. Her urine examination demonstrated pyuria and microscopic hematuria. Extended-spectrum $\beta$-lactamase-producing (ESBL) Escherichia coli was detected in her urine culture and appropriate antibiotherapy was given. A $25 \times 15 \mathrm{~mm}$ opacity at bony pelvis was observed on her plain $\mathrm{X}$-ray radiography of the abdomen and a $3 \times 2 \mathrm{~cm}$ bladder stone was seen on ultrasound (Fig. 1). With these findings, cystoscopy was performed under spinal anesthesia. A stone surrounding the polypropylene mesh at the right base of the bladder wall was observed. Stone over the mesh was fragmented using both holmium-YAG laser (Sphinx ${ }^{\circledR}$ LISA laser, $80 \mathrm{~W}, 2120$ $\mathrm{nm}, 0.5-4.5 \mathrm{~J})$ and pneumatic lithotripter (Elmed ${ }^{\circledR} V$ ibrolith). After the stone was removed, we attempted to cut the mesh with endoscopic scissors, but this was unsuccessful due to the strength of the mesh. Transurethral resection of the mesh using resectoscope was also tried, but this approach failed as well. Finally, a holmium laser was used and all the pieces of mesh were easily removed (Fig. 2). An 18 French (Fr) Foley catheter was kept on free drainage for three days. At the eight-month followup, the patient was dry and had no urinary complaints

\section{Discussion}

Mini-sling, a third-generation tape, was introduced to avoid the complications of TVT or TOT in the treatment of SUI. 


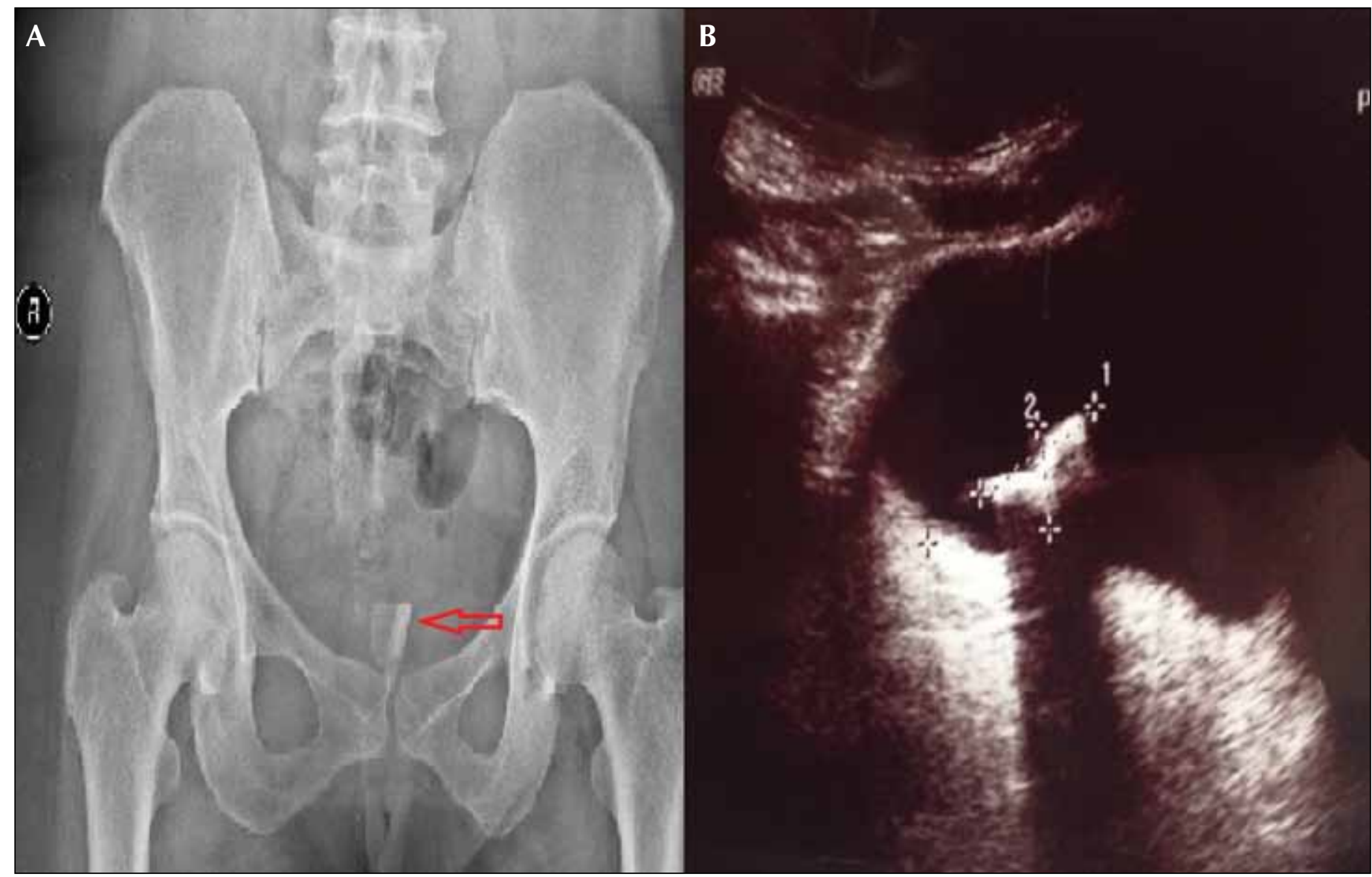

Fig. 1. (A) Plain X-ray radiography and (B) ultrasound images of the calcified mesh.

However, some undesired complications, such as bladder perforation and stone formation over the mesh, may occur with this procedure. There are only a few publications about these injuries. Previous studies indicate that most of these injuries occurred with TVT-Secur. ${ }^{5}$ There are only a few bladder injuries reported with the MiniArc ${ }^{\circledR}$ sling in the current literature. ${ }^{6}$ In our case, a needleless device had been used in another centre for the procedure. To our knowledge, there is no published data on bladder perforation using this device.

Experience is required to avoid bladder perforation in incontinence surgeries. These patients may present with multiple symptoms, including dysuria, hematuria, and irritative or obstructive urinary symptoms after surgery. ${ }^{7}$ Bladder injuries should be considered in patients presenting with such symptoms, even if needleless surgery was performed. The use of cystoscopy in such cases is useful to confirm the perforation diagnosis.

Removing an intravesical mesh from the bladder is a clinical dilemma. In recent studies, endoscopic scissors, resectoscope, and laser have been used..$^{5,8}$ In our case, we tried to cut the intravesical mesh with endoscopic scissors, but the mesh was too strong. Resectoscope was also attempted, but the intravesical sling could not cut using this approach. Finally, holmium laser was used and the mesh was removed easily. Doumouchtsis et al investigated mesh or suture excisions from the bladder using holmium laser and suggested this procedure as a first-line treatment in these cases. ${ }^{9}$

\section{Conclusion}

Bladder perforation and stone formation over the intravesical mesh after a mini-sling surgery may occur, even when a needleless device is used. In patients who experience recurring urinary system infections with irritative or obstructive urinary symptoms following a mini-sling procedure, bladder perforation should considered and cystoscopy performed to confirm the diagnosis. An endoscopic approach using holmium laser is effective in such a complication.

Competing interests: The authors report no competing personal or financial interests.

This paper has been peer-reviewed. 


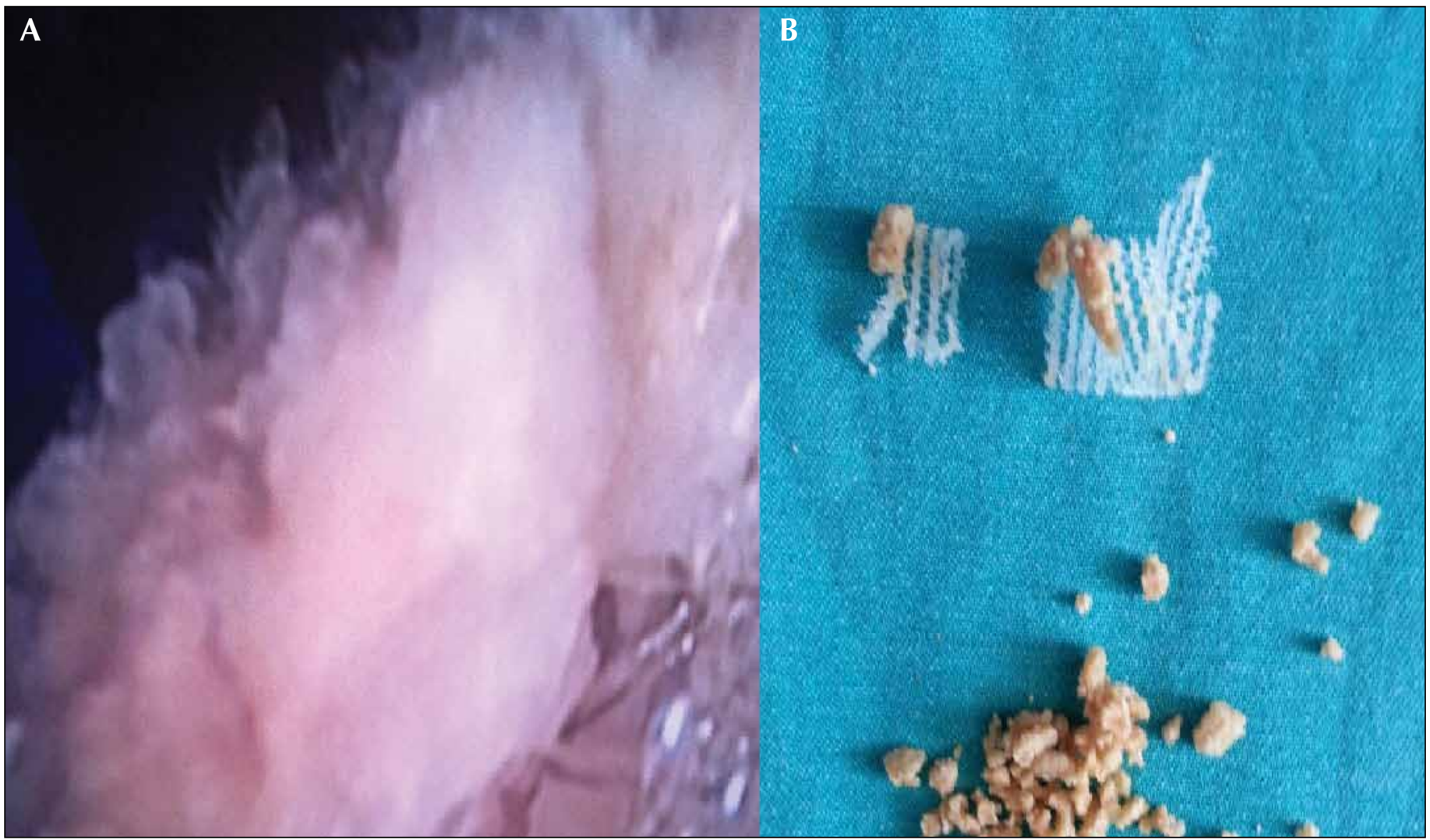

Fig. 2. (A) Cystoscopic view of the bladder stone formed over the mesh; and (B) excised mesh and stones.

\section{References}

1. Chapple CR. Retropubic suspension surgery for incontinence in women. In: Wein AJ, Kavoussi LR, Novick AC, et al., eds. Campbell-Walsh Urology. 10th ed. Philadelphia, PA: Elsevier Saunders; 2011 :chap 71.

2. Ulmsten U, Henriksson L, Johnson $P$, et al. An ambulatory surgical procedure under local anesthesia for treatment of female urinary incontinence. Int Urogynecol J Pelvic Floor Dysfunct 1996;7:81-5. https://doi.org/10.1007/BF01902378

3. Delorme E. Transobturator urethral suspension: mini-invasive procedure in the treatment of stress urinary incontinence in women. Prog Urol 2001;11:1306-13.

4. Coskun B, Lavelle RS, Alhalabi F, et al. Mini-slings can cause complications. Int Urogynecol I 2015 26:557-62. htrps://doi.org/10.1007/s00192-014-2530-7

5. Zivanovic I, Kociszewski J, Eberhard J, et al. Bladder perforation after TVT-Secur procedure: How secure is TVT-Secur? Arch Gynecol Obstet 2014;289:131-4. https://doi.org/10.1007/s00404-013-2968-8
6. Gauruder-Burmester A, Popken $G$ The mini-arc sling system in the treatment of female stress urinary incontinence. Int Braz I Urol 2009;35: 34-41. https://doi.org/10.1590/S1677-55382009000300010

7. Jo DJ, Lee YS, Oh TH, et al. Outcomes of transurethral removal of intravesical or intraurethral mesh following midurethral sling surgery. Korean J Urol 2011;52:829-34. https://doi.org/10.4111/ kju.2011.52.12.829

8. Feiner B, Auslender R, Mecz $Y$, et al. Removal of an eroded transobturator tape from the bladder using laser cystolithotripsy and cystoscopic resection. Urology 2009;73:681.e15-6

9. Doumouchtsis SK, Lee FY, Bramwell D, et al. Evaluation of holmium laser for managing mesh/suture complications of continence surgery. BJU Int 2011;108:1472-8. https://doi.org/10.1111/i.1464410X.2010.10012.x

Correspondence: Dr. Mustafa Karalar, Department of Urology, Afyon Kocatepe University, Afyonkarahisar, Turkey; drkaralar@yahoo.com 